\title{
超声场中 $\mathrm{Sn}-38.1 \% \mathrm{~Pb}$ 共晶合金的动态凝固
}

\author{
翟薇, 洪振宇, 解文军, 魏炳波 ${ }^{*}$ \\ 西北工业大学应用物理系, 西安 710072 \\ * 联系人, E-mail: bbwei@nwpu.edu.cn \\ 2010-03-22 收稿, 2010-06-09 接受 \\ 国家自然科学基金资助项目(50971105)
}

\begin{abstract}
摘要 $\mathrm{Sn}-38.1 \% \mathrm{~Pb}$ 共晶合金在频率为 $35 \mathrm{kHz}$ 的超声场中发生凝固时, 随着试样高度 $H$ 的降低, 声场对宏观偏析的抑制效果更加显著, 球状共晶团在凝固组织中所占体积分数相应增大. 当 $H$ 等于声波在合金熔体内的波长 $\lambda$ 时, 超声场能够扩大初生( $\mathrm{Sn}$ )相分布区域，缩小 $\mathrm{Sn}-\mathrm{Pb}$ 共晶和初 生 $(\mathrm{Pb})$ 相分布区域. 同时, 初生 $(\mathrm{Sn})$ 相晶粒显著细化, 生长方式发生“枝晶-等轴晶”的转变. 对于 $H=\lambda / 2$ 和 $\lambda / 4$ 的合金试样, 超声场能够促进熔体形核, 降低合金整体过冷度. 分析表明, 超声场 中的空化效应抑制了熔体过冷, 声流促进共晶生长界面前沿的温度场和浓度场呈空间对称分布, 使 $\mathrm{Sn}-\mathrm{Pb}$ 共晶以三维对称方式生长.
\end{abstract}

关键词

超声场

共晶生长

球状共晶团

空化效应

声流
超声场中合金熔体的凝固规律是材料物理领域 的重要研究课题 ${ }^{[1-8]}$. 在合金凝固过程中, 超声波的 传播不仅引人声场能量, 还能够引发一系列非线性 效应, 如声空化、声流效应, 最终可能改变合金凝固 组织形貌及性能 ${ }^{[1 \sim 4]}$. 研究表明, 超声场对于凝固过 程的作用机制主要表现为细化晶粒 ${ }^{[4 \sim 6]}$ 、超声除气 ${ }^{[7]}$ 和抑制偏析 ${ }^{[8,9]}$ 等几个方面. Campbell ${ }^{[2]}$ 总结了众多研 究者的研究结果, 指出超声的施加方式、声强和频率 是影响超声对凝固过程作用效果的关键因素. 然而, 超声场能够在多大试样尺寸范围内对凝固组织起到 有效调控作用这一重要问题通常被研究者所忽略. 实际上，超声波在合金熔体中传播时，能够在固液界 面上发生反射、透射和散射等多种现象. 同时, 凝固 所生成的固相颗粒与熔体之间的相对运动能够引发 声能量的迅速耗散 ${ }^{[10]}$, 从而声强会随着传播距离的 增加而发生衰减, 并且空化和声流等非线性效应也 会随之减弱 ${ }^{[11]}$, 这就有可能使超声场对于凝固组织 的作用仅局限于一定的尺度范围内. 目前有关这方 面的研究还不多见.

另一方面, 从研究对象的选取来看, 目前大多数
的研究集中于超声作用下的单相枝晶生长研究，而 对于涉及到二相或多相共生生长的共晶合金在超声 作用下的凝固特征研究相对较少. $\mathrm{Sn}-\mathrm{Pb}$ 共晶体系是 常见的低熔点二元共晶型合金. 本文以 $\mathrm{Sn}-38.1 \% \mathrm{~Pb}$ 共晶合金为研究对象，研究超声场中不同高度的凝 固试样沿声波传播方向的组织演变特征, 实验确定 声场作用的有效尺度范围，并探讨超声作用下的共 晶生长机制.

\section{1 实验}

实验在带有压电式超声换能器的凝固装置中进 行. $\mathrm{Sn}-38.1 \% \mathrm{~Pb}$ 共晶合金由高纯 $\mathrm{Sn}(99.9 \%)$ 和 $\mathrm{Pb}(99.9 \%)$ 熔配而成. 根据声波在此液态合金内部传 播的波长 $\lambda=66 \mathrm{~mm}$, 选取了 $H=\lambda, \lambda / 2$ 和 $\lambda / 4$ 三种不同 高度的合金试样，置于尺寸为 $10 \mathrm{~mm} \times 13 \mathrm{~mm} \times 80 \mathrm{~mm}$ 的 $\mathrm{Al}$ 坩埚内. 实验过程中, 用电阻炉加热熔化试样, 熔体温度由 $\mathrm{NiCr}-\mathrm{NiSi}$ 热电偶测定. 对于 $H=\lambda$ 和 $\lambda / 2$ 的合金试样, 利用 3067 型高精度记录仪分别对试样 顶部和底部的冷却曲线进行记录. 对于 $H=\lambda / 4$ 的试样, 由于高度较低, 仅记录合金试样中部的冷却曲线. 超 
声换能器的频率为 $35 \mathrm{kHz}$, 利用直径为 $9.6 \mathrm{~mm}$ 的圆 形端面变幅杆将声波从顶部导人合金熔体内部. 变 幅杆在插人合金熔体前进行预热，当熔体温度降至 共晶温度 $T_{\mathrm{E}}$ 以上约 $100 \mathrm{~K}$ 时, 对合金熔体施加纵波 直至试样完全凝固. 实验过程中从坩埚顶部连续通 人 $\mathrm{Ar}$ 气流，以防止合金氧化. 对于不同高度的试样， 超声换能器的输人电压值和谐振状态均保持不变, 声源处声强值约为 $12 \mathrm{~W} / \mathrm{cm}^{2}$.

实验结束后, 将凝固试样纵剖后镶嵌抛光, 并利 用 Zeiss Axiovert 200 MAT 光学显微镜和 FEI Sirion 200 扫描电子显微镜对凝固组织进行分析表征.

\section{2 结果与讨论}

\section{1 超声场中 $\boldsymbol{H}=\lambda$ 试样的凝固过程}

图 1(a)是高度 $H=\lambda$ 的合金试样分别在静态条件 和超声作用下的冷却曲线. 可以看出, 凝固过程是无 定向条件的均匀凝固. 静态条件下试样顶部和底部 的过冷度 $\Delta T$ 分别为 9 和 $10 \mathrm{~K}$. 施加超声场后, 试样 顶部的过冷度降低到 $5 \mathrm{~K}$, 而底部的过冷度为 $9 \mathrm{~K}$. 显然，超声场有效降低了合金顶部的过冷度，而对于 合金底部过冷度影响较小，说明超声场对于合金过 冷度的改变作用随声波传播距离的增加而减弱.

图 2 为静态条件和超声作用下 $\mathrm{Sn}-38.1 \% \mathrm{~Pb}$ 合金 凝固组织的生长形态分布图. 静态条件下, 沿重力方 向凝固组织形态表现出明显的区域特征, 如图 2(a) (c)所示. 区域 I 中, 大量的初生 $(\mathrm{Sn})$ 相以枝晶方式生 长, 枝晶主干平均长度达到 $262 \mu \mathrm{m}$, 少量的 $\mathrm{Sn}-\mathrm{Pb}$ 规 则共晶分布于 $(\mathrm{Sn})$ 枝晶间隙处. 区域 II 中的凝固组织 完全为规则的 $\mathrm{Sn}-\mathrm{Pb}$ 层片共晶, 层片共晶平均间距为 $2.0 \mu \mathrm{m}$. 在区域 III 中, 许多 $(\mathrm{Pb})$ 枝晶分布在 $\mathrm{Sn}-\mathrm{Pb}$ 规 则共晶基底上. 且越靠近试样底部, ( $\mathrm{Pb}$ )枝晶越粗
大，分布越密集. 区域 III 内的 $(\mathrm{Pb})$ 枝晶平均主干长度 达到 $308 \mu \mathrm{m}$, 二次枝晶臂间距为 $28 \mu \mathrm{m}$. 如图 3 所示, I , II 和 III 这三个典型区域分别占试样总高度的 $22 \%$, $13 \%$ 和 $65 \%$.

施加超声场后, 试样沿着声传播方向的生长形 态分布由 $I^{\prime} ， I^{\prime}$ 和 III' 这三个区域组成，如图 2(d) (f) 所示. 这三个区域的高度与静态条件下相比发生明 显变化，分别占试样总高度的 $51 \%, 6 \%$ 和 $43 \%$. 显然， 超声场改变了合金试样内部的宏观偏析形式，即扩 大了初生 $(\mathrm{Sn})$ 相的分布区域，而缩小了 $\mathrm{Sn}-\mathrm{Pb}$ 共晶以 及初生 $(\mathrm{Pb})$ 相的分布区域. 进一步对比区域 $\mathrm{I}$ 和 $\mathrm{I}^{\prime}$ 发 现，区域 $I^{\prime}$ 内的凝固组织生长形态发生了显著改变. 初生 $(\mathrm{Sn})$ 相不再以树枝晶方式生长, 而是呈球状等轴 晶均匀弥散分布在共晶基底上，其平均晶粒尺寸显 著减小到 $36 \mu \mathrm{m}$. 可见，超声场对于区域 $\mathrm{I}^{\prime}$ 内的初生 $(\mathrm{Sn})$ 晶粒具有明显的细化作用. 同时，在区域 I'中还 出现了很多以球状方式生长的共晶团, 如图 2(d)所 示. 与静态条件下相比, 区域 I' 中的共晶组织不再 以平行的规则层片方式生长. 从图 2(e)中可以看出, 共晶层片生长方向发生偏移或是层片发生了碎断, 规则程度明显下降。但是，平均层片共晶间距与静 态条件下相比并没有发生明显变化. 对于区域 $\mathrm{III}^{\prime}$ 中 的 $(\mathrm{Pb})$ 枝晶, 枝晶主干长度和二次枝晶臂间距均与 静态条件下对应区域内基本相同。不难看出，在该 强度声源下，超声对微观凝固组织的改变作用仅限 于靠近声源的区域 I'和区域 II'内，而对区域 III'作用 不明显，说明超声波在合金的凝固过程中传播时衰 减迅速，对微观凝固组织的有效作用范围小于 $\lambda$ 而接 近 $\lambda / 2$.

静态条件下，在 $\mathrm{Sn}-38.1 \% \mathrm{~Pb}$ 共晶合金凝固过程 中, $(\mathrm{Pb})$ 相首先从整个过冷熔体中形核并生长. 由于 密度远大于熔体密度, 初生 $(\mathrm{Pb})$ 相会在重力作用下下
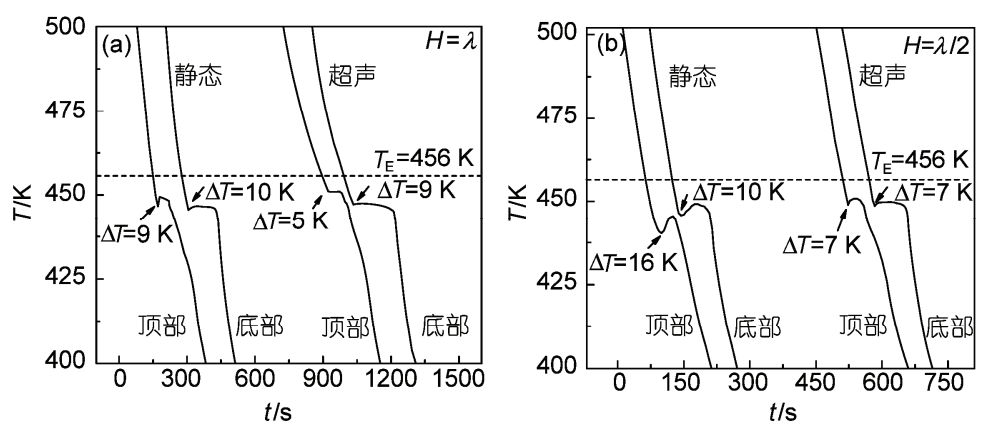

图 $1 \mathrm{Sn}-38.1 \% \mathrm{~Pb}$ 合金冷却曲线 (a) $H=\lambda$; (b) $H=\lambda / 2$; (c) $H=\lambda / 4$

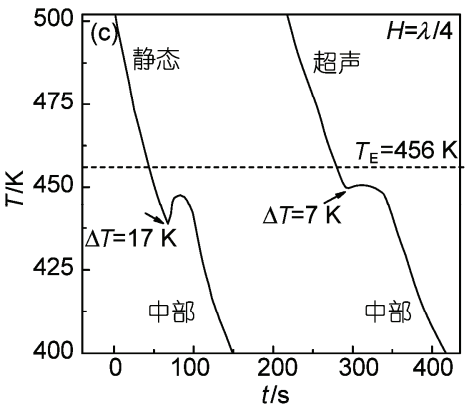




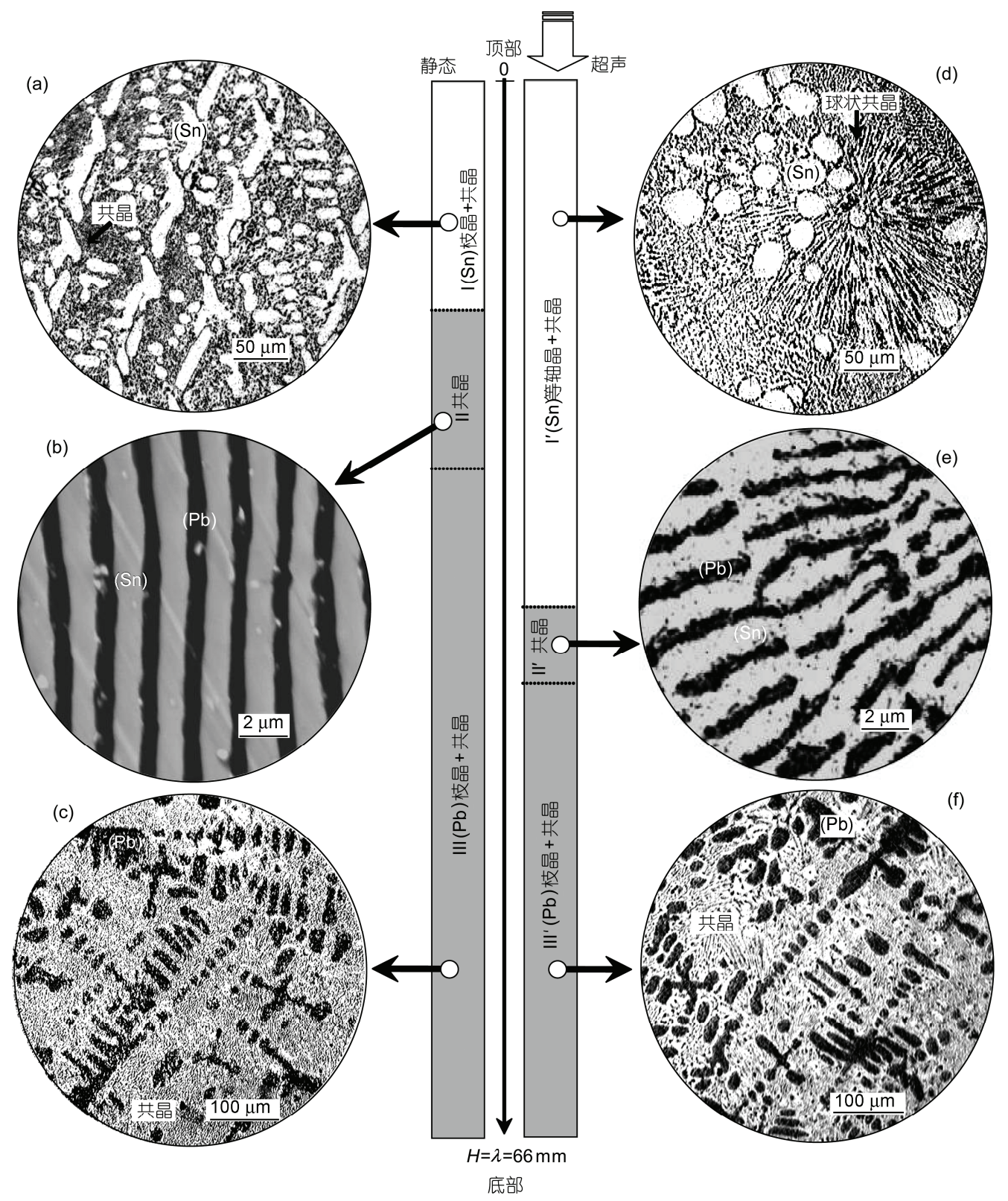

图 $2 \mathrm{Sn}-38.1 \% \mathrm{~Pb}$ 共晶合金在 $\boldsymbol{H}=\lambda$ 情况下组织形态分布图 (a) (c) 静态条件; (d) (f) 超声作用

沉至试样下部. 随后, 密度较小的 $(\mathrm{Sn})$ 枝晶从富 $(\mathrm{Sn})$ 熔体中析出并上浮至试样上部, 而剩余熔体中 $(\mathrm{Sn})$ 相 与 $(\mathrm{Pb})$ 相以共晶方式协同生长, 最终形成了分层的宏 观偏析组织. 超声作用下, 试样内部凝固组织的宏观 偏析形式在一定程度上取决于声波在合金凝固过程 中的有效作用范围. 在靠近声源的区域 I'内, 超声作 用使合金熔体过冷度减小, 从而初生 $(\mathrm{Pb})$ 相的析出受 到抑制, 熔体趋于以共晶方式协同生长. 而在区域 III'
内, 熔体过冷度较大, $(\mathrm{Pb})$ 相以枝晶方式析出后在重 力作用下下沉. 由于远离声源, $(\mathrm{Pb})$ 相在向下的运动 和生长过程几乎不受超声场影响. 此时, (Sn)相以初 生枝晶方式从区域 III'内的剩余富 $(\mathrm{Sn})$ 熔体中析出, 并 在重力场中浮力驱动对流作用下上浮. 当初生 $(\mathrm{Sn})$ 相 上浮进人有效的超声场作用范围后, 声流能够部分 抵消浮力驱动对流, 阻碍或减慢初生 $(\mathrm{Sn})$ 相的上浮, 从而使初生 $(\mathrm{Sn})$ 相能够在超声场的有效作用区域内 
趋于均匀分布. 这是超声作用下 $H=\lambda$ 的 $\mathrm{Sn}-\mathrm{Pb}$ 共晶合 金试样宏观偏析形式发生改变的原因.

\section{2 超声场中 $H=\lambda / 2$ 和 $\lambda / 4$ 试样的凝固组织}

由于超声场不能对 $H=\lambda$ 内全部凝固组织的微观 生长形态产生明显的改变作用, 实验将合金试样尺 寸降低到 $H=\lambda / 2$ 和 $\lambda / 4$, 以研究较小高度试样在超声 场作用下的共晶生长特征. 图 1(b) 和(c)是这两种高 度试样在静态条件和超声作用下的冷却曲线. 静态 条件下, $H=\lambda / 2$ 试样顶部和底部的形核过冷度 $\Delta T$ 分别 为 16 和 $10 \mathrm{~K}$. 超声作用下, 顶部和底部的过冷度均 减小至 $7 \mathrm{~K}$. 同样, 对于 $H=\lambda / 4$ 的合金试样, 声场作 用下的合金形核过冷度也由静态条件下的 17 减小到 $10 \mathrm{~K}$. 显然，超声场降低了这两种高度的合金试样的 整体过冷度, 促进了熔体形核.

静态条件下, 随着合金试样高度的降低, 凝固组 织的宏观偏析程度大大降低. 不同高度合金试样内 部各种凝固形态所占的高度分数 $h_{f}$ 由图 3 给出. 对于 $H=\lambda / 2$ 的合金试样, 凝固组织主要由 $\mathrm{Sn}-\mathrm{Pb}$ 共晶组织 组成, 只有少数细小的初生 $(\mathrm{Pb})$ 枝晶零星分布在试样 下部约占试样总高度 $30 \%$ 的区域内. 加人超声场后, 仅在试样最底部 $7 \%$ 的高度区域内有极少量的 $(\mathrm{Pb})$ 枝 晶存在, 而完全的 $\mathrm{Sn}-\mathrm{Pb}$ 共晶组织占试样总高度的 $93 \%$. 对于 $H=\lambda / 4$ 的合金试样, 静态条件下, $(\mathrm{Pb})$ 相分 布区域减小到 $18 \%$, 而超声作用下, $(\mathrm{Pb})$ 相区域消失, 试样内部为 $100 \%$ 的共晶组织. 不难发现, 随着试样 高度的降低, $\mathrm{Sn}-38.1 \% \mathrm{~Pb}$ 合金中凝固组织的宏观偏 析现象在超声场中得到有效抑制.

图 4 给出了 $H=\lambda / 2$ 的合金试样内部 $\mathrm{Sn}-\mathrm{Pb}$ 共晶生 长形态. 静态条件下, $\mathrm{Sn}-\mathrm{Pb}$ 共晶以规则层片方式生 长, 平均层片间距约为 $1.4 \mu \mathrm{m}$, 如图 4(a)所示. 超声 作用下, 共晶组织由多个紧密相连的球状共晶团组 成, 共晶团直径为 100 400 $\mu \mathrm{m}$, 如图 4(b) 所示. 对共 晶团内部组织的形貌观察发现, 主要存在如图 4(c)和 (d)两种典型的形态. 在图 4(c)中所示的共晶团中心, 极其细小的 $(\mathrm{Sn})$ 相和 $(\mathrm{Pb})$ 相均匀弥散分布, 形成不规 则共晶, 而规则层片共晶则依附于不规则共晶呈放 射状外延生长. 这种组织特征表明, 共晶生长的形核 位置应该是共晶团中央, 并且不规则共晶组织先于 规则层片共晶生成. 在图 4(d)中, $\mathrm{Sn}-\mathrm{Pb}$ 共晶以层片 方式由共晶团中心向外部呈辐射状生长. 与静态条 件下的 $\mathrm{Sn}-\mathrm{Pb}$ 共晶层片相比, 超声场中所形成的规则
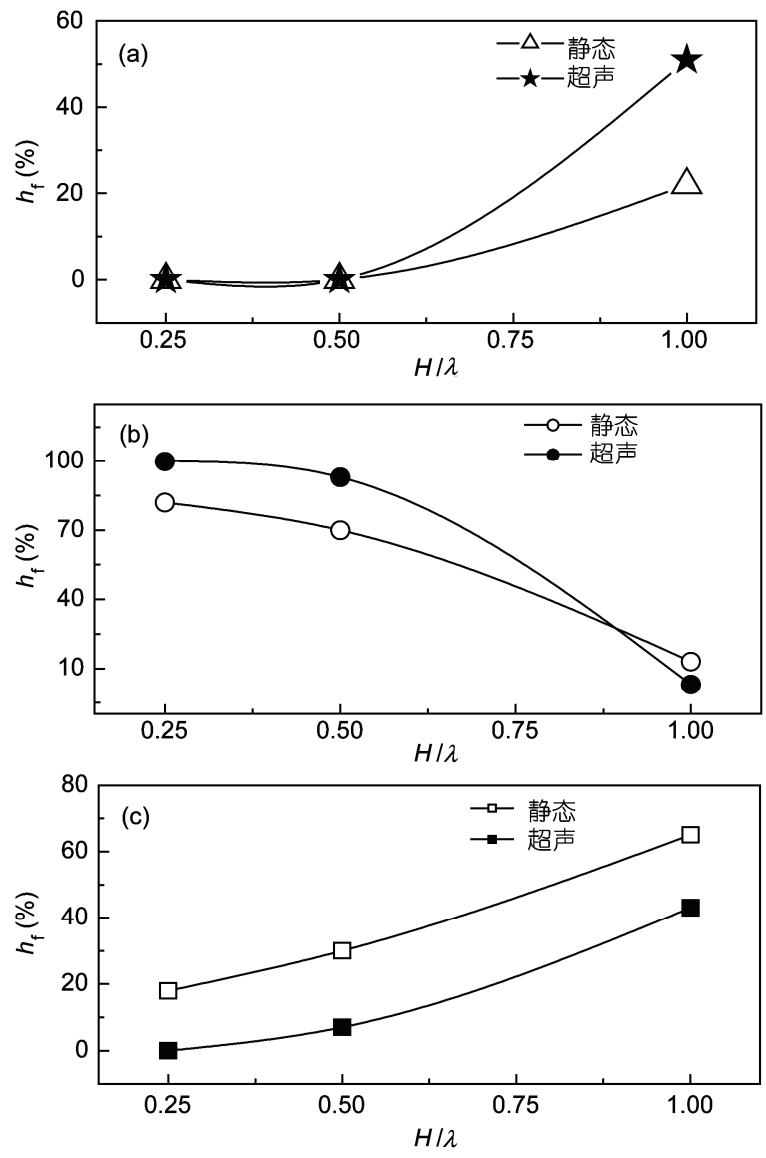

图 3 三种组织形态区域所占高度分数随试样高度的变化

(a) (Sn)相+共晶; (b) 共晶; (c) (Pb)相+共晶

层片平均间距约为 $5.0 \mu \mathrm{m}$, 明显大于静态条件下的 间距. 声场对于 $\mathrm{Sn}-\mathrm{Pb}$ 共晶具有明显的粗化作用.

$H=\lambda / 4$ 的合金试样在超声作用下的共晶组织生 长形态与 $H=\lambda / 2$ 试样十分相似, 这里不再赘述.

\section{3 超声场作用下的共晶生长机制}

从上述凝固组织特征可以看出, 在微观尺度上, 超声场对于 $\mathrm{Sn}-\mathrm{Pb}$ 共晶凝固机制的影响主要表现在 以下两个方面: (1) 降低合金熔体整体过冷度, 促进熔 体形核; (2) 促使凝固试样内部形成大量球状共晶团.

由于静态条件和超声作用下合金试样整体过冷 度均较小, 所以异质形核是主要的形核机制. 熔体异 质形核过程中需要克服的形核激活能 $\Delta G$ 可以表示 为 ${ }^{[12]}$

$$
\Delta G=\frac{16 \pi \sigma^{3} T_{\mathrm{m}}^{2}}{3 \Delta H_{\mathrm{m}}^{2} \Delta T^{2}} \cdot f(\theta)
$$




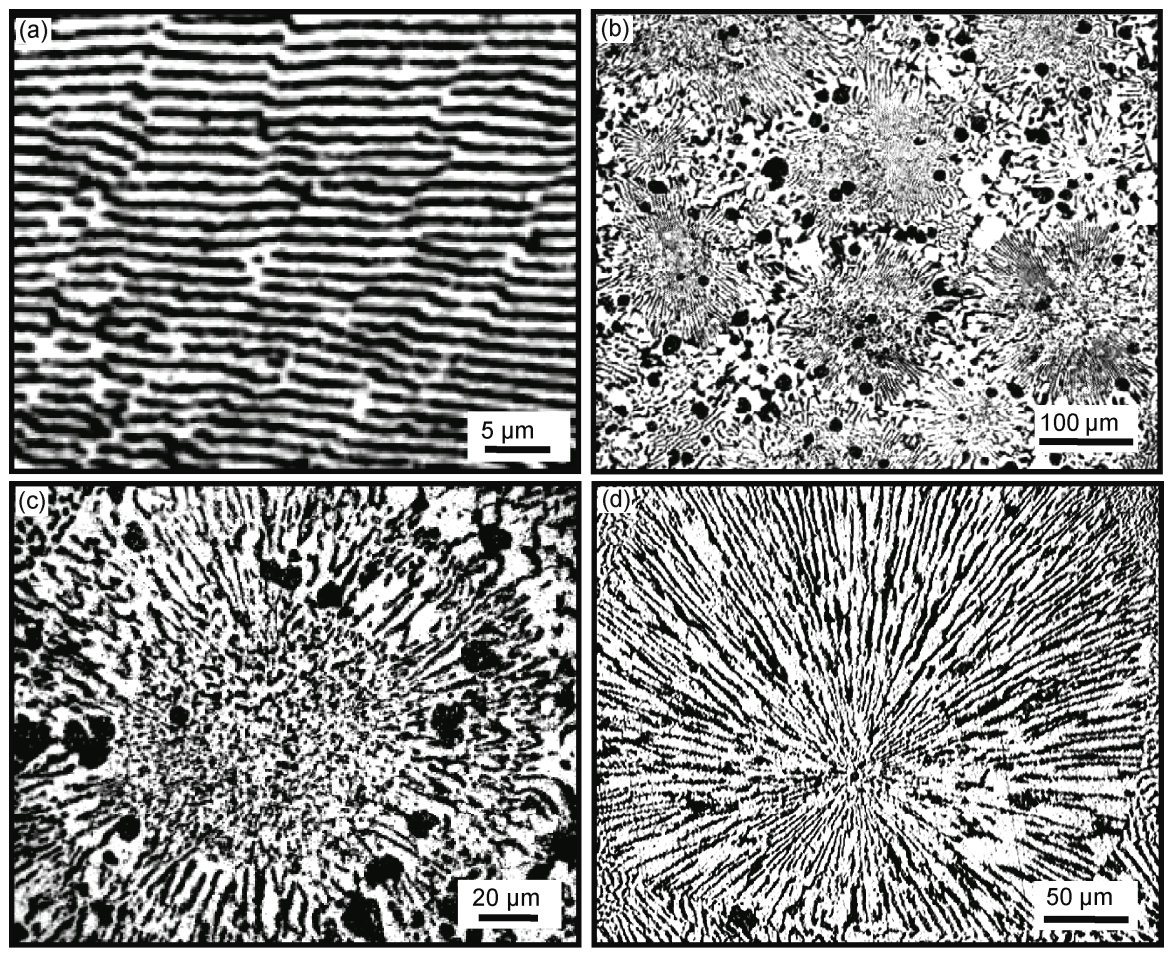

图 $4 \quad \mathrm{Sn}-38.1 \% \mathrm{~Pb}$ 合金在 $H=\lambda / 2$ 时的共晶组织形态

(a) 静态条件下的规则层片共晶; (b) 超声作用下形成的大量共晶团; (c) 不规则共晶的球状共晶团; (d) 规则层片共晶的球状共晶团

这里, $\sigma$ 为液固界面自由能; $T_{\mathrm{m}}$ 是合金熔点; $\Delta H_{\mathrm{m}}$ 表示 液固转变所引起的焓变化; $\theta$ 为熔体中晶胚对异质质点 的润湿角, $0^{\circ} \sim 180^{\circ}, f(\theta)$ 是润湿角因子, $f(\theta)=(2+\cos \theta)$ $\times(1-\cos \theta)^{2} / 4$. 合金熔体发生凝固至少需要一个晶 核, 即必须满足 $I \cdot V \cdot t \geqslant 1$. 其中 $I$ 是形核率, $V$ 是合金 熔体的体积, $t$ 是凝固时间. 根据图 1(c)中测定的静态 条件下的凝固时间 $32 \mathrm{~s}$, 可计算得静态条件下 $H=\lambda / 4$ 合金试样中 $f(\theta) \leqslant 0.05143$, 即 $\theta \leqslant 43^{\circ}$.

空化效应是声场促进熔体形核的主要原因之一. 当超声波在熔体中传播时, 可以在熔体中的微区内 产生很多的微小孔穴和气泡. 这些孔穴和气泡在声 场的激发下不停地振荡长大. 当气泡长大到一定程 度时, 会发生破裂并在气泡壁处产生 1 5 GPa 的瞬时 高压, 对熔体形核过程产生重要影响.

另一方面, 虽然经过净化处理, 合金熔体中还是 不可避免地存在一些微小的杂质. 当超声波在合金 熔体中传播时, 能够显著改善这些异质质点与熔体 之间的润湿程度, 降低晶胚与异质质点之间的润湿 角, 从而有可能引发大量异质点, 促使凝固在小的过 冷度下发生.

空化效应发生过程中, 合金熔点 $T_{\mathrm{m}}$ 随局域压强
$P$ 的变化关系由 Clausius-Clapeyron 方程 ${ }^{[12]}$ 给出:

$$
T_{\mathrm{m}}=T_{\mathrm{E}}+\frac{T_{\mathrm{E}} \Delta V}{\Delta H_{\mathrm{m}}}\left(P-P_{0}\right) .
$$

上式中, $T_{\mathrm{E}}$ 是 $\mathrm{Sn}-38.1 \% \mathrm{~Pb}$ 共晶合金在一个大气压 $P_{0}$ 下的熔点, $\Delta V$ 是液固转变所引起的体积变化. 根据(2) 式计算了空化效应所产生的压强 $P$ 对于合金熔体局 域过冷度 $\Delta T_{\text {Local }}$ 的影响规律, 如图 5(a)所示. 当空化 产生 1 5 GPa 的微小局域高压时, 空化处的局域过冷 度提高 29 145 K, 从而能够对形核过程产生重要影 响. 以 $H=\lambda / 4$ 合金试样为例, 由(1)和(2)式计算了不 同压强和润湿角作用下的 $(\mathrm{Sn})$ 相和 $(\mathrm{Pb})$ 相形核激活能. 计算中所用到的物理参数取自文献[13], 并在表 1 中 列出, 计算结果分别示于图 5(b)和(c)中. 从图中可以 看出, $(\mathrm{Pb})$ 相和 $(\mathrm{Sn})$ 相形核激活能均随压强的增高和 润湿角的降低而显著减小. 在相同的润湿角下, 空化 产生的 GPa 级的局域高压能够使两共晶相的形核激 活能下降约 2 个量级. 由此可见, 在超声场中, 空化 部位所承受的微区高压能够提高熔体局域过冷从, 从而显著降低形核激活能, 是十分有利于形核的位 置. 而在相同的压强下, 当润湿角从 $43^{\circ}$ 降低到 $1^{\circ}$ 

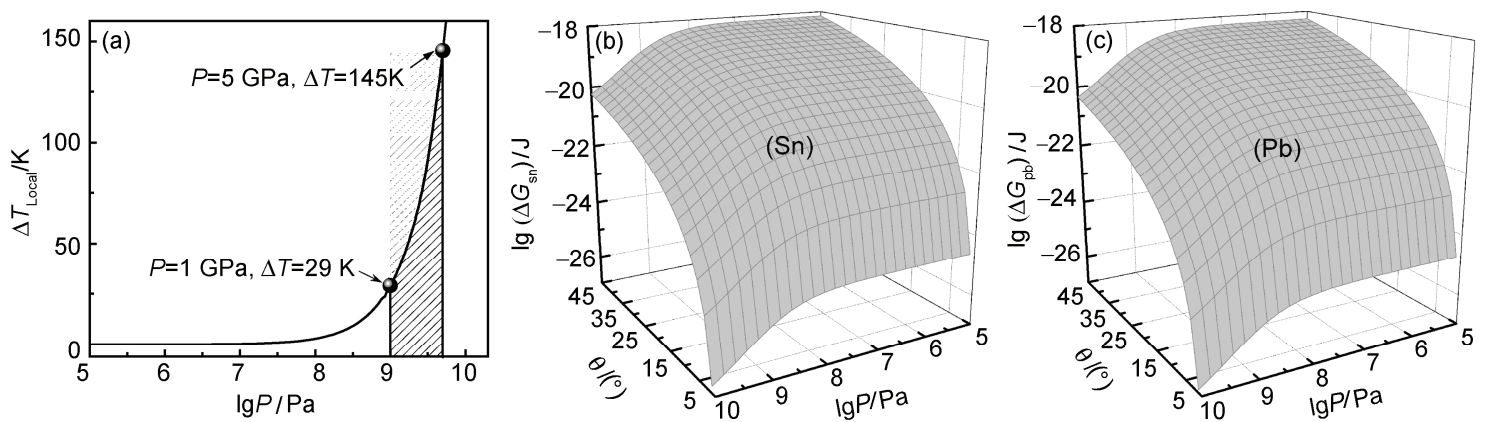

图 5 合金熔体的局域过冷度和形核激活能

(a) 不同压强下的局域过冷度; (b) (Sn)相形核激活能随压强和润湿角的变化; (c) (Pb)相形核激活能随压强和润湿角的变化

表 1 计算所用物理参数 ${ }^{[13]}$

\begin{tabular}{cc}
\hline 物理参数 & 数值 \\
\hline 共晶温度 $T_{\mathrm{E}} / \mathrm{K}$ & 456 \\
$(\mathrm{Sn})$ 相熔化焓 $\Delta H_{m-\mathrm{Sn}} / \mathrm{J} \cdot \mathrm{m}^{-3}$ & $4.41 \times 10^{8}$ \\
$(\mathrm{~Pb})$ 相熔化焓 $\Delta H_{m-\mathrm{Pb}} / \mathrm{J} \cdot \mathrm{m}^{-3}$ & $3.21 \times 10^{8}$ \\
$(\mathrm{Sn})$ 相熔化时相对体积变化 $\Delta V_{\mathrm{Sn}}(\%)$ & 2.3 \\
$(\mathrm{~Pb})$ 相熔化时相对体积变化 $\Delta V_{\mathrm{Pb}}(\%)$ & 3.5 \\
$(\mathrm{Sn})$ 相固液界面能 $\sigma_{\mathrm{Sn}} / \mathrm{J} \cdot \mathrm{m}^{-2}$ & $5.8 \times 10^{-2}$ \\
$(\mathrm{~Pb})$ 相固液界面能 $\sigma_{\mathrm{Pb}} / \mathrm{J} \cdot \mathrm{m}^{-2}$ & $4.3 \times 10^{-2}$ \\
\hline
\end{tabular}

时，两共晶相的形核激活能下降约 6 个量级. 形核激 活能的降低意味着熔体形核能力的增强. 所以，空化 效应和声场对润湿角的减小作用是超声场中液态合 金总体过冷度降低的重要原因.

在形核后的共晶生长过程中，超声场中的声流 效应促进了共晶生长界面前沿的温度场和浓度场呈 空间对称分布，使共晶团以三维对称方式生长. 如果 空化效应引发的局域过冷度足够大，该处的合金熔 体以不规则共晶方式生长 ${ }^{[14,15]}$. 同时, 熔体内液相的 流动能够以空化泡为中心形成环流 ${ }^{[16]}$. 这种环流能 够保证共晶生长界面前沿温度场和浓度场的空间对 称性，使凝固界面以球状推进. 随后，由于固液界面
处凝固潜热的释放, 使过冷度减小, 产生规则层片共 晶并依附于不规则共晶生长, 最终形成如图 4(c) 所示 的共晶团. 也就是说，空化产生的局域大过冷和声流 引发的空间对称分布的浓度场和温度场是形成这一 组织形态的两个动力学条件. 如果空化引发的局域 过冷度不足够大, 或是共晶生长起源于异质晶核时, 在声流引发的空间对称的温度场和浓度场作用下, $\mathrm{Sn}-\mathrm{Pb}$ 共晶以规则层片方式辐射生长, 形成如图 4(d) 的球状共晶团. 上述声场作用下两种类型球状共晶 团的形成过程如图 6 所示. 需要指出的是, 在规则共 晶生长过程中, 液相的流动能够加速液固界面前沿 共晶两相之间原子的互扩散，增加扩散距离，从而共 晶层片与静态条件下相比，发生显著的粗化.

\section{3 结论}

采用不同高度试样，研究了超声场中 $\mathrm{Sn}-38.1 \%$ $\mathrm{Pb}$ 共晶合金的动态凝固过程，主要得到以下结论：

(1) 超声场能够改变凝固组织宏观偏析形式. 当 合金试样高度 $H=\lambda$ 时，超声场扩大了初生 $(\mathrm{Sn})$ 相分布 区域，而缩小了 $\mathrm{Sn}-\mathrm{Pb}$ 共晶和初生 $(\mathrm{Pb})$ 相分布区域. 同

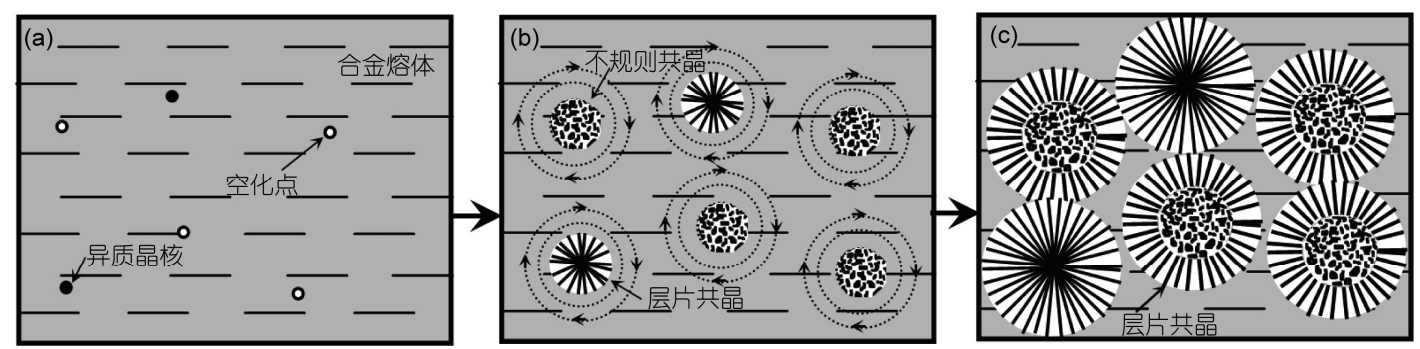

图 6 超声场中球状共晶团形成示意图

(a) 空化效应引发的优先形核和声场激发的异质晶核; (b) 局域深过冷处的不规则共晶生长和局域小过冷处的层片共晶生长; (c) 两种球状共晶团 
时, 初生 $(\mathrm{Sn})$ 相生长形态发生“树枝晶-等轴晶”的转 变, 且晶粒尺寸显著细化. 当 $H$ 降低到 $\lambda / 2$ 和 $\lambda / 4$ 时, 超声场能够显著抑制甚至消除凝固组织内部的宏观 偏析.

（2）超声作用下的 $H=\lambda / 2$ 和 $\lambda / 4$ 凝固试样中得到 两种典型的 $\mathrm{Sn}-\mathrm{Pb}$ 球状共晶团, 一种是规则层片依附 于共晶团中心不规则共晶外延生长, 另一种是整个 共晶团内部以规则层片方式呈辐射状生长. 空化效
应产生足够高的局域过冷度，以及声流促使共晶生 长前沿温度场和浓度场呈空间对称分布, 是形成前 一种共晶团组织形貌的两个动力学条件.

（3）超声场中，空化效应产生的局域高压所引 发的熔体局域大过冷, 以及声波传播过程中对熔体 内晶胚与异质质点润湿程度的增强, 均能引发合 金熔体形核，使熔体整体过冷度比静态条件下显著 降低.

致谢＼cjkstart实验过程中得到王飞、代富平和王伟丽等同事的有益帮助，在此深表感谢.

\section{参考文献}

1 Abramov O V. Action of high intensity ultrasound on solidifying metal. Ultrasonics, 1987, 25: 73-82

2 Campbell J. Effects of vibration during solidification. Int Mater Rev, 1981, 2: 71-103

3 Abramov V, Abramov O, Bulgakov V, et al. Solidification of aluminum alloys under ultrasonic irradiation using water-cooled resonator. Mater Lett, 1998, 37: 27-34

4 Han Y F, Shu D, Wang J, et al. Microstructure and grain refining performance of Al-5Ti-1B master alloy prepared under high-intensity ultrasound. Mater Sci Eng A, 2006, 430: 326-331

5 Jian X, Xu H, Meek T T, et al. Effect of power ultrasound on solidification of aluminum A356 alloy. Mater Lett, 2005, 59: 190-193

6 翟薇, 洪振宇, 解文军, 等. 超声场中 $\mathrm{Ag}-\mathrm{Cu}-\mathrm{Sb}$ 三元共晶的生长机制. 中国科学 $\mathrm{G}$ 辑: 物理学 力学 天文学, 2007, 37: 367一-375

7 Li X T, Li T J, Li X M, et al. Study of ultrasonic melt treatment on the quality of horizontal continuously cast Al-1\%Si alloy. Ultrason Sonochem, 2006, 13: 121-125

8 翟薇, 洪振宇, 解文军, 等. 高频声场作用下 $\mathrm{Pb}-\mathrm{Sb}$ 过共晶合金凝固过程研究. 科学通报, 2006, 51: 2476一 2480

9 Puga $\mathrm{H}$, Teixeira $\mathrm{J} \mathrm{C}$, Barbosa J, et al. The combined effect of melt stirring and ultrasonic agitation on the degassing efficiency of $\mathrm{AlSi}_{9} \mathrm{Cu}_{3}$ alloy. Mater Lett, 2009, 63: 2089-2092

10 苏明旭, 蔡小舒, 徐峰, 等. 超声衰减法测量悬浊液中颗粒粒度和浓度. 声学学报, 2004, 29: 440一444

11 刘清梅, 龚永勇, 侯旭, 等. 侧部导人超声处理对共晶 Al-Si 合金凝固特性的影响. 中国有色金属学报, 2007, 17: 307一-312

12 Kurz W, Fisher D J. Fundamentals of Solidification. 3rd ed. Switzerland: Trans Tech Publications Ltd, 1998. 30-36

13 Brandes E A. Smithells Metals Reference Book. 6th ed. England: Butterworth \&Co Ltd, 1983. 8: 1-54

14 Dai F P, Xie W J, Wei B. Spherical ternary eutectic cells formed during free fall. Phil Mag Lett, 2009, 89: 170-177

15 Han X J, Wang N, Wei B. Rapid eutectic growth under containerless condition. Appl Phys Lett, 2002, 81: 778—780

16 Chow R, Blindt R, Chivers R, et al. A study on the primary and secondary nucleation of ice by power ultrasound. Ultrasonics, 2005, 43: 227 $-230$ 